\title{
Management
}

\section{of Treatment-Resistant Panic Disorder}

\author{
Giampaolo Perna, $M D, P h D^{1,2,3, *}$ \\ Daniela Caldirola, MD, $P h D^{1}$
}

\author{
Address \\ *,1Department of Clinical Neurosciences, Villa San Benedetto Menni Hospital, \\ FoRiPsi, Hermanas Hospitalarias, Albese con Cassano, 22032, Como, Italy \\ Email: pernagp@gmail.com \\ ${ }^{2}$ Department of Psychiatry and Neuropsychology, Faculty of Health, Medicine and \\ Life Sciences, Maastricht University, Maastricht, 6200, The Netherlands \\ ${ }^{3}$ Department of Psychiatry and Behavioral Sciences, Leonard Miller School of \\ Medicine, Miami University, Miami, FL, 33136-1015, USA
}

Published online: 24 0ctober 2017

(C) The Author(s) 2017. This article is an open access publication

This article is part of the Topical Collection on Anxiety, Obsessive Compulsive, and Related Disorders

Keywords Panic disorder · Treatment-resistant · Pharmacotherapy · Cognitive behavioral therapy $\cdot$ Personalized treatment

\begin{abstract}
Purpose of Review Management of treatment-resistant (TR) panic disorder (PD) is an unresolved issue. In this paper, we provide a brief summary of previous findings, an updated (2015-2017) systematic review of pharmacological/non-pharmacological studies, and our personal perspective on this topic.

Recent Findings We found a very limited number of recent findings. Quetiapine extendedrelease augmentation has not been found to be beneficial, in comparison to placebo, in non-responders to previously recommended pharmacotherapy. In non-responders to cognitive behavioral therapy (CBT), switching to paroxetine/citalopram has been found to be more effective than continuing CBT. Acceptance and commitment therapy (ACT) has shown some improvement in patients' resistance to previous psychological/ pharmacological interventions compared with a waiting-list condition.

Summary Previous and recent findings regarding the treatment of TR PD suffer from several methodological limitations. Available studies provide insufficient evidence to support the use of medications alternative to the recommended medications; the efficacy of ACT needs confirmation with more rigorous methodology. Prolonged pharmacotherapy may produce significant improvement in patients with unsatisfactory response to short-term pharmacotherapy, while switching to pharmacotherapy may help non-responders to CBT. We discuss our personal perspective on the definition of "treatment resistance" as it relates to PD and provide personalized intervention strategies to increase favorable clinical
\end{abstract}


outcomes based on our clinical expertise and review of experimental studies on the pathophysiology of PD.

\section{Introduction}

Panic disorder (PD) is a chronic, debilitating anxiety disorder that exhibits a lifetime prevalence of $3-4 \%$ in the general population [1]. PD is associated with psychiatric/medical comorbidity; significant impairment of daily functioning, work performance, and quality of life; and relevant social costs [2, 3].

The core features of PD are recurrent, unexpected panic attacks (PAs) characterized by sudden, intense fear/discomfort episodes, with a surge of somatic symptoms such as chest pain, palpitations, dyspnea, and breathlessness. Patients also exhibit anticipatory anxiety and/or maladaptive changes in behavior related to PAs. Most subjects with PD fear or avoid multiple situations in which PAs can occur (i.e., agoraphobia) [4].

Several medications, as well as cognitive behavioral therapy (CBT), are effective for PD. Medications include selective serotonin reuptake inhibitors (SSRIs), serotonin-norepinephrine reuptake inhibitor (SNRI) venlafaxine, tricyclic antidepressants (TCAs), and benzodiazepines. Among these, SSRIs and venlafaxine are considered to be first-line treatment agents because of their efficacy and favorable side effect profiles $[2,5,6$, $7 \bullet, 8]$. Despite these treatment options, in short-term clinical trials, $17-64 \%$ of participants with PD did not adequately respond to pharmacotherapy and continued to have PAs and/or avoidance symptoms [9•], and in clinical settings, approximately $20-40 \%$ of patients did not achieve full remission with the recommended drugs or CBT [10-12]. As medications based on novel mechanisms are far from being implemented in clinical settings $[7 \bullet]$, optimizing existing treatment options appears to be the most viable strategy in the near future for increasing the rate of successful outcomes in PD. Unfortunately, limited empirical findings are available to guide clinicians and indicate next-step strategies for individuals with treatment-resistant (TR) PD. Current guidelines do not offer clear indications to choose from available options for treatment-refractory patients, such as dosage modifications for current pharmacotherapy, switching within or between classes of medications or to other treatment modalities, or augmentation strategies for the use of additional medications or other treatment modalities $[2,5]$. Clinical decision-making support algorithms and/or evidence-based predictors of treatment response are lacking, thereby negatively affecting the potential to tailor treatments according to each patient's characteristics [13•]. Considering these unresolved issues, the aims of our paper are as follows: (1) to give a brief summary of the "state-of-the-art" evidence on this topic based on the most recent reviews, (2) to undertake an updated (2015-2017) systematic review of pharmacological and non-pharmacological studies in patients with TRPD, and (3) to comment on the available findings and provide our point of view on this topic.

\section{The "state-of-art" in TRPD: previous findings on definition and treatment options}

Currently, there is no unequivocal definition of TRPD. Very heterogeneous criteria have been used across different studies, making interpretation of the results uncertain. Some authors have defined patients with TR as those with PD who do not respond to at least one [14,15] or two [16-18] 8-week treatments with adequate doses of drugs recognized as effective for PD or those who do not respond to a standard course of CBT. Other authors have nominated shorter ( 6 weeks) $[19,20]$ or longer (12-16 weeks) [21-23] time periods. In addition to heterogeneity in the number and duration of treatments, discrepancies in the psychometric assessment of panic-phobic symptoms/illness severity, as well as 
in the "cut-off" used to define response/remission, were present across different studies, thereby increasing the variability of the definition of TR.

Finally, some studies have used a more comprehensive evaluation of clinical response than others. Some authors have proposed that patients with TR be defined as those with PD who, after 6 months of recommended treatments, fail to achieve almost complete resolution of PAs, anticipatory anxiety, panicrelated phobias, functional/social impairment [assessed separately or incorporated into the Panic Disorder Severity Scale (PDSS)], general anxiety, and depressive symptoms secondary to PD [24-26]. In conclusion, a consensus on the definition of TR is still lacking.

The most recent, comprehensive systematic review on pharmacological/ non-pharmacological interventions in patients with TRPD, covering studies published from 1980 to the beginning of 2015 [ $9 \bullet]$, included 11 studies, only two of which were double-blind randomized controlled trials (RCTs) [16, 19]. A small, preliminary RCT suggested that 4-week pindolol (7.5 mg/day) adjunctive treatment to fluoxetine $(20 \mathrm{mg} /$ day $)$ was significantly superior to placebo in improving panic/anxiety symptoms in patients with PD who were resistant to at least two previous treatments with antidepressants and an 8-week trial with fluoxetine $(20 \mathrm{mg} /$ day) [16]. In the other RCT, in a small sample of patients with PD who had not achieved remission after 6 weeks of treatment with sertraline (up to $100 \mathrm{mg} /$ day) or escitalopram (up to $15 \mathrm{mg} /$ day), 6 subsequent weeks of a higher dosage of the same medications (150-200 mg/day or 20$30 \mathrm{mg} /$ day, respectively) did not improve panic/anxiety symptoms in comparison to adjunctive placebo [19].

The other nine open studies (five pharmacological and four CBT trials) found preliminary indications of some efficacy of monotherapy with reboxetine or olanzapine and an augmentation strategy for recommended medications with pindolol, divalproex sodium, aripiprazole, and olanzapine or 12 group sessions of CBT. However, several methodological flaws, such as open design, small sample size, heterogeneous criteria for the definition of TRPD and outcome measures, and concurrent use of multiple pharmacological agents in augmentation studies, undermined the overall reliability of the results $[9 \bullet]$.

\section{The update: a systematic review of pharmacological/non- pharmacological studies in patients with TRPD}

\section{Methods}

This updated systematic review was performed according to the PRISMA guidelines [27]. A database search of peer-reviewed scientific literature, written in English, was conducted using PubMed, PsycINFO, and Embase, from January 1 , 2015 to July 1, 2017. The following search terms were used: panic AND ( ${ }^{*}$ resistant OR * resistance OR non-respond* OR nonrespond* OR non respond* OR refractory). We also used the reference lists of relevant studies and pertinent review articles to gain access to additional literature. Among 290 records identified in the search, three studies were included in the review (Fig. 1, PRISMA flow diagram). Each step of the search and selection procedures was independently performed by the two authors, and inconsistency in the results were discussed and resolved before proceeding. 


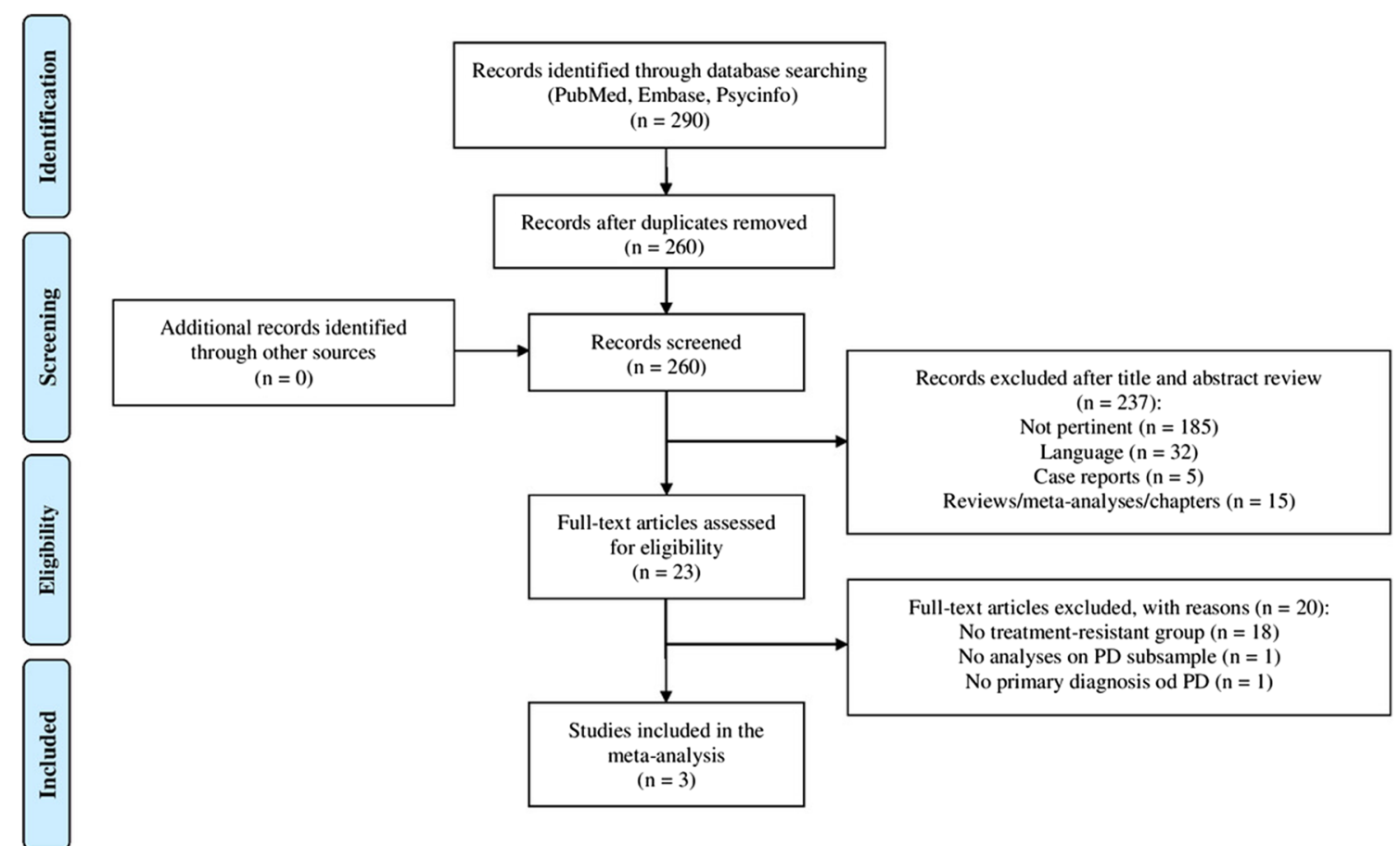

Fig. 1. PRISMA flow diagram of the study selection process.

Studies were included in the review if they comprised participants aged $\geq 18$ years, with a primary diagnosis of TRPD (all definitions of treatment resistance were accepted) with or without agoraphobia according to DSM-IV/ DSM-IV-TR [28, 29], DSM V [4], ICD 9/ICD-9-CM [30, 31], or ICD-10 [32] criteria; pharmacological and/or non-pharmacological treatments (all types of experimental design were accepted); validated self-reported and/or clinicianadministered psychometric scales as efficacy outcome measures; if the studies provided separate results in subgroups with PD when multiple anxiety disorders were studied; and if full texts were available. We excluded case reports, commentaries, letters, editorials, reviews, meta-analyses, book chapters, and conference abstracts.

We included three studies in this review, which are described in detail in Table 1.

A proof-of-concept RCT evaluated the efficacy of adjunctive quetiapine extended release (XR) (flexible doses) compared with adjunctive placebo (8 weeks) in a small sample of SSRI/SNRI-resistant patients with a principal diagnosis of PD with or without agoraphobia [33•]. Most had comorbid psychiatric conditions. The methods used to define SSRI/SNRI resistance varied, as it was determined either historically or prospectively depending on the conditions of the patients during intake of SSRI/SNRI. During the trial, improvement of panic symptoms (as the primary outcome) and secondary outcome measures were observed in the whole sample, but no significant differences between quetiapine XR and placebo were found. In addition, no 


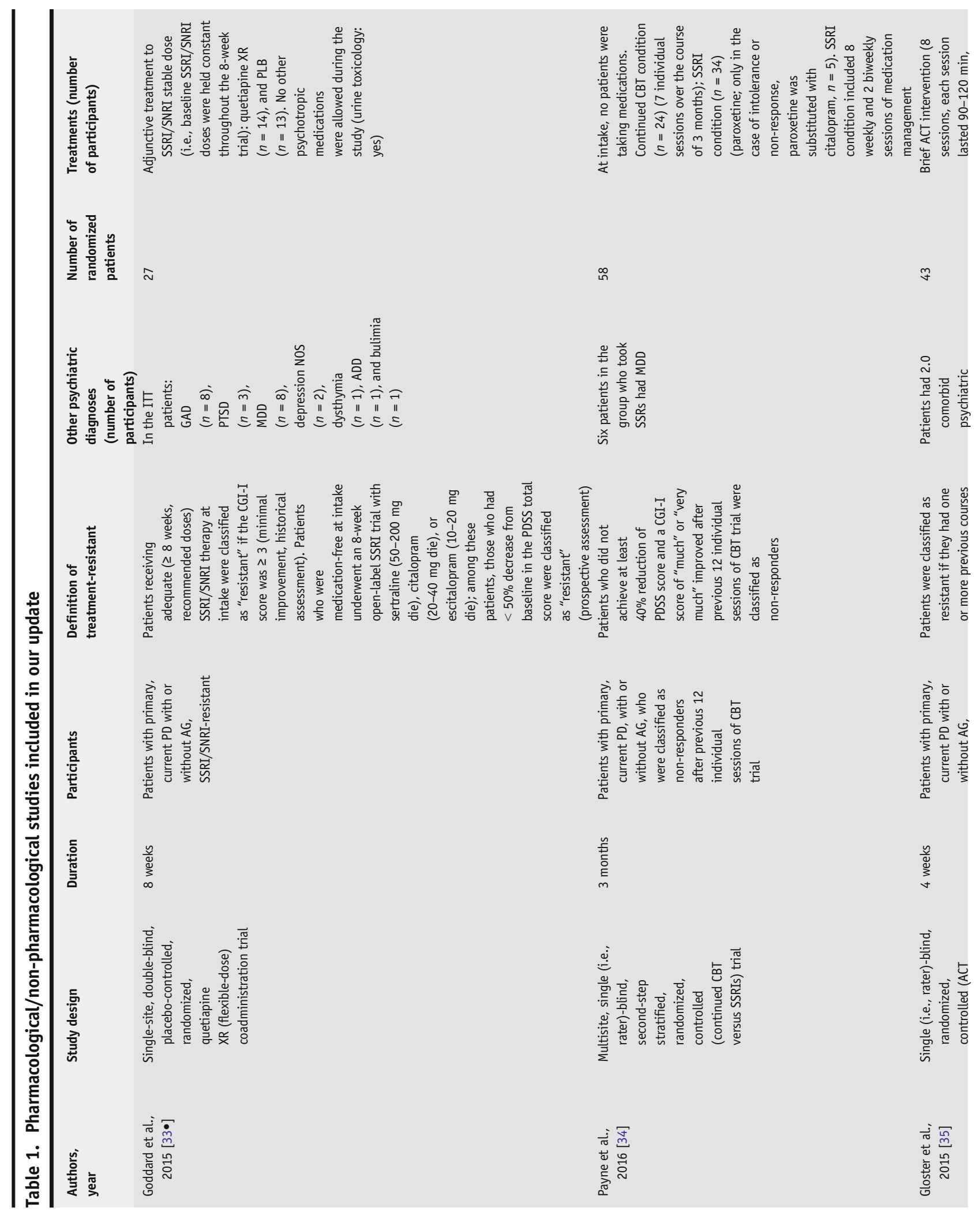




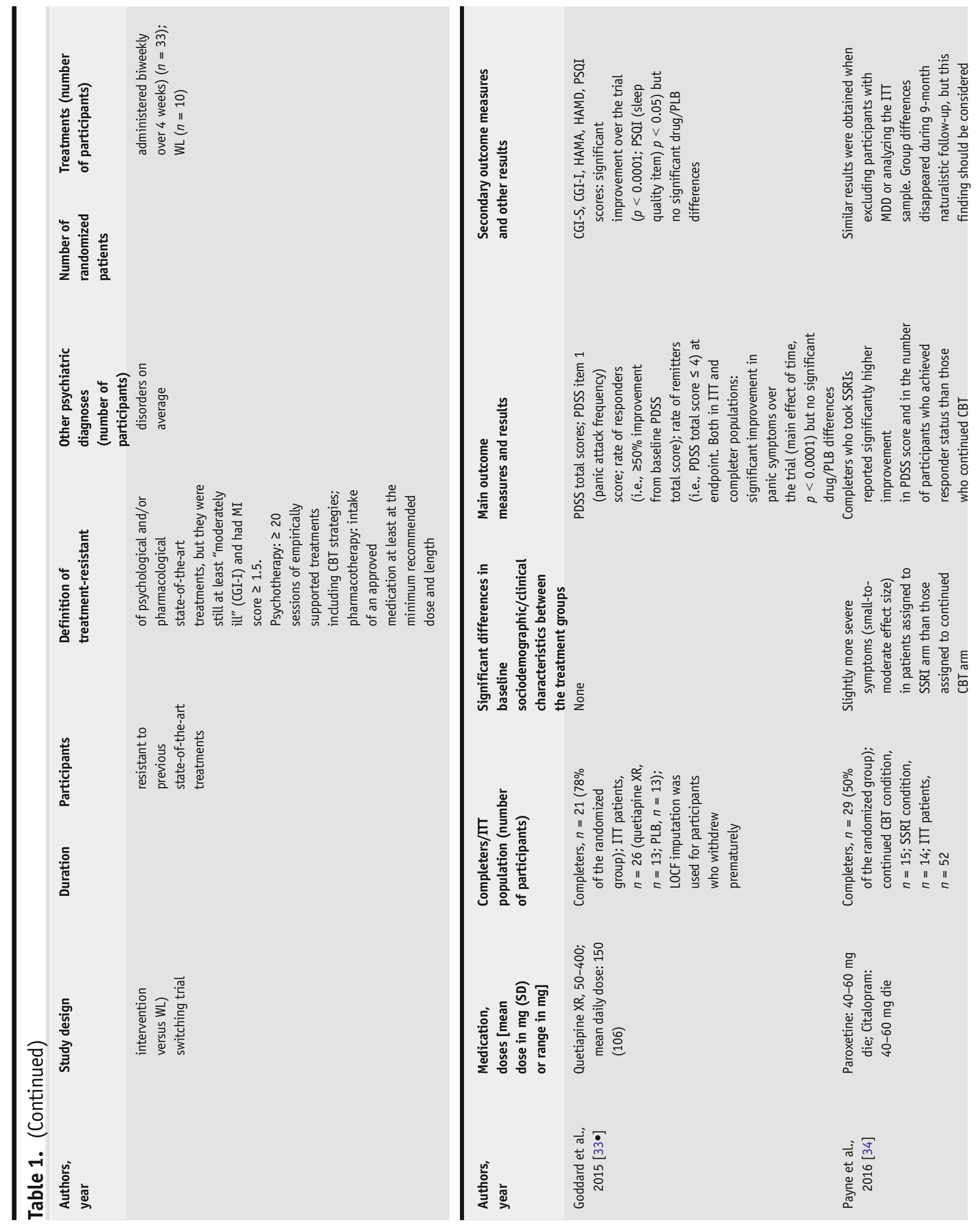




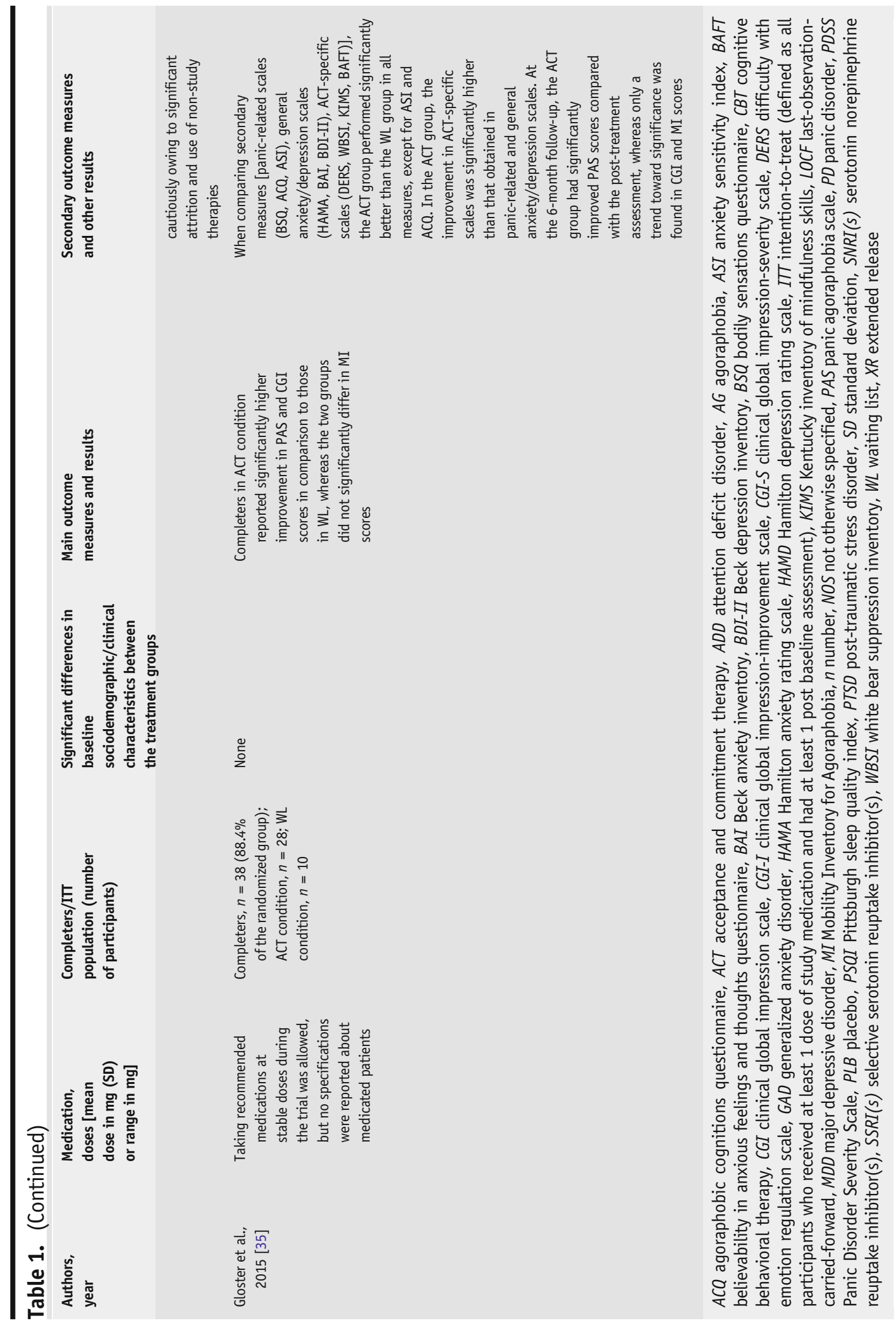




\section{Conclusions}

significant differences were found in terms of treatment-related side effects. This study had sufficient statistical power to detect large effect sizes, whereas it was unable to detect small-to-moderate effect sizes.

A 3-month second-step, randomized, controlled trial conducted by Payne et al. [34] examined whether a sequenced treatment consisting of switching to pharmacotherapy (paroxetine or citalopram) versus continuing CBT provided benefits in a small sample of patients with PD who had not responded to a trial of 12 individual sessions of CBT (without medications). At the end of the treatment period, the participants who took SSRIs reported significantly lower panic symptoms in comparison to those who continued CBT.

A proof-of-principle, randomized, waiting-list (WL) controlled 4-week trial [35] compared the effectiveness of a brief, intensive psychological intervention with acceptance and commitment therapy (ACT) to WL in a small sample of patients with PD who were resistant to previous psychological and/or pharmacological interventions. At the end of the treatment period, there was some improvement found in the ACT group in comparison to the WL group in terms of panic-phobic symptoms, global illness severity, general anxiety/depressive symptoms, and fear related to bodily sensations, whereas no significant differences were found between the two groups in terms of agoraphobia (as measured using the Mobility Inventory for Agoraphobia), anxiety sensitivity, or agoraphobic cognitions. Patients who were taking medications were admitted to the trial if they agreed not to change the doses during the study. However, the authors did not report data on the number of medicated patients nor on the doses/classes of those medications.

\section{Comments on the available findings}

There are insufficient previous findings on treatment options for patients with PD who remain symptomatic after receiving initial recommended interventions, and disappointingly, our updated review yielded a very limited number of additional studies.

Previous indications of some efficacy of monotherapy with reboxetine or olanzapine and augmentation strategies with recommended medications with pindolol, divalproex sodium, aripiprazole, or olanzapine [9•] should be considered with caution because of the major methodological limitations of the studies. In addition, the suggestion that reboxetine monotherapy is effective for patients with TRPD [ $9 \bullet$ ] does not seem promising, considering the results of other experimental studies. Indeed, a previous study conducted by our group showed that reboxetine, a selective noradrenaline reuptake inhibitor, was significantly less effective than paroxetine (an SSRI) in decreasing the frequency of PAs induced by inhalation of $35 \%$ carbon dioxide $\left(\mathrm{CO}_{2}\right) / 65 \%$ oxygen $\left(\mathrm{O}_{2}\right)$ gas mixture in patients with PD [36]. Similarly, in a randomized single-blind study, we found a greater effect of paroxetine on clinical PAs than reboxetine, whereas no differences were found in anticipatory anxiety and avoidance [37]. These findings suggest that modulation of the serotonergic system is more relevant for PAs, the "core" symptoms of PD, than the modulation of the noradrenergic system, thereby casting doubts on the clinical usefulness of switching to reboxetine, at least in patients who still have PAs after receiving a standard treatment. With regard to second-generation antipsychotics (SGAs), in contrast 
to some previous open trials described by Freire et al. [9•], the recent randomized placebo-controlled study included in our update [33•] found that a quetiapine XR augmentation strategy failed to improve either primary (panic symptoms) or secondary outcome measures in a sample of SSRI/SNRI-resistant patients with PD. Although the study had limitations, such as its mixed and unstandardized method of defining SSRI/SNRI resistance and a small sample size with a wide variety of psychiatric comorbidities, its negative results discouraged the use of quetiapine XR augmentation in patients with TRPD. Overall, considering the uncertain anti-panic efficacy and the possibly unfavorable side effect profile of SGAs, these medications do not seem to be viable treatment options in the realm of both treatment-sensitive and TR panic, particularly in patients with a primary or sole diagnosis of PD (for a specific review on this topic, see Perna et al. [38•]).

No recent studies have addressed the issue of the optimal doses/period of time of recommended pharmacotherapy before considering a patient with PD to be a non-responder. However, RCT conducted by Simon et al. $[9 \bullet, 19]$ suggested that maintaining medium recommended doses of sertraline/escitalopram for additional 6 weeks in patients who did not achieve remission during the previous 6 weeks of treatment can facilitate additional, significant improvement, whereas increase to the maximum doses did not do so. These results are consistent with other studies that found significant improvement in patients with PD over the course of long-term pharmacotherapy beyond that obtained with short-term treatment with the same medications [39-41]. Overall, these indications suggest that at least in patients who show some improvement during the first 6-8 weeks of pharmacotherapy, more prolonged treatments can be used before considering these patients to be TR.

Opposite findings have been reported for CBT in a recent RCT conducted by Payne et al. included in our update [34]. This RCT suggested that extending the duration of a standard course of CBT in patients deemed to be non-responders was significantly less effective than switching to paroxetine or citalopram. These results are in line with those of a double-blind RCT that found adjunctive paroxetine to have significantly higher efficacy than placebo in patients with PD who were unsuccessfully treated with CBT alone [42].

Finally, the recent preliminary study by Gloster et al. [35] has suggested some efficacy of a brief, very intensive ACT intervention in patients with TRPD. However, several limitations, such as a small sample size, lack of an active therapy as a control condition, and lack of information about concomitant medications, do not allow reliable conclusions to be made.

Overall, available findings on this topic suffer from several shortcomings, such as limited sample size; significant heterogeneity in treatment resistance criteria and/or outcome measures; and insufficient use of psychometric tools that separately assess unexpected/expected PAs, anticipatory anxiety, and phobic avoidance, thereby negatively affecting the potential to define which aspects of PD do and do not respond to therapy. Large-scale, systematic studies that include evidence-based predictors of response, as well as algorithm-based longitudinal investigations (such as STAR*D for depression), are still lacking.

In conclusion, at present, available findings are insufficient to support the use of medications alternative to those recommended for the clinical management of patients with TRPD. Similarly, the recent suggestion on the efficacy of 
ACT for patients with TRPD needs confirmation by studies with more rigorous methodology.

Some studies have indicated that more prolonged pharmacotherapy may facilitate further clinical improvement in patients with unsatisfactory response to short-term pharmacotherapy. Recent findings in relation to patients with PD who did not adequately respond to CBT suggest that switching to SSRIs or adjunctive SSRI treatment may be more effective than continuing CBT alone.

Finally, no recent studies have investigated the usefulness of CBT in pharmacotherapy non-remitted patients with $\mathrm{PD}$, whereas a previous review found significant efficacy of adjunctive CBT as a next-step strategy in this population of patients [43].

In this section, we would like to offer some personal views on this topic based on our clinical expertise and some experimental evidence.

In our opinion, clinical practice may offer a more encouraging picture of TRPD than clinical trials. When dealing with patients with PD who apparently did not obtain optimal results from various therapies, careful examinations based on in-depth knowledge of panic mechanisms often reveal to clinicians different factors that may explain the persistence of clinical symptoms rather than an actual "resistance" to treatment.

As a first step, medical conditions (e.g., cardiopulmonary conditions and hyperthyroidism) or use of stimulant substances (e.g., caffeine) that can provoke/worse panic symptoms should be excluded. The presence of untreated, current, comorbid psychiatric disorders should also be evaluated and addressed with specific interventions, which are out of scope of this review.

With regard to primary $\mathrm{PD}$, in many cases, an unsatisfactory response to treatment may be due to an incomplete clinical recognition of the different psychopathological domains active in the patient, resulting in poor personalization of therapy and subsequent apparent resistance to treatment. Indeed, discriminating between the psychopathological organizing element and secondary clinical events is crucial to choosing the most appropriate intervention. In the realm of $\mathrm{PD}$, unexpected PAs are the "core symptoms" of the disorder, whose recurrence influences the subsequent development of anticipatory anxiety and/or maladaptive changes in behavior, including agoraphobia; similarly, other clinical phenomena such as hypochondria, depressive symptoms, benzodiazepine/ alcohol abuse, and social phobia are often secondary to the presence and persistence of panic symptoms [4]. Therefore, the primary therapeutic intervention should aim to completely prevent panic recurrence, including full blown PAs, limited-symptom PAs, sub-threshold panic symptoms, and sensations of physical discomfort, with the achievement of a full sense of physical wellbeing. It should be noted that even when obvious PAs are not present, many patients with PD experience sensation of an imminent occurrence of PAs, without actual clinical manifestations and/or sub-continuous somatic sensations in their daily life, such as dyspnea, tachycardia, or dizziness, that make them feel unfit. All these phenomena may be manifestations of physiologic somatic system instability, mainly involving cardiorespiratory and balance systems, which are implicated in the pathophysiology of panic [36, 44]. If persistent, these manifestations, particularly the sub-threshold symptoms/physical discomfort that often remain 
unrecognized in clinical practice, can maintain anticipatory anxiety/phobic avoidance as defensive active mechanisms, as well as other secondary clinical phenomena, thereby mimicking resistance to treatment. For example, patients who are apparently "resistant" because of their continuing agoraphobia after a standard course of CBT may in fact have unrecognized active panic symptoms that require a re-evaluation of the therapeutic choice. Consistent with this, the findings discussed above show that in patients with PD who have not adequately responded to CBT, switching to SSRIs or an adjunctive SSRI treatment may be more effective than continuing CBT alone $[34,42]$ and may be related to pharmacotherapy-induced improvement of panic symptoms. Similarly, the indications that more prolonged pharmacotherapy may facilitate significant improvement in patients who did not fully respond to recommended short-term therapy $[19,39-41]$ suggest that adjunctive weeks may allow medications to exert broader effects on the multiple clinical manifestations of panic vulnerability.

Patients with PD who continue to experience panic symptoms despite receiving recommended pharmacotherapy should be evaluated as to whether their prescribed medications are actually targeted to their symptom profiles; if it is not the case, changing the compound should be considered. Indeed, although current guidelines for PD describe recommended medications as a group with similar effects and comparative studies between compounds are scant, preliminary experimental evidence has suggested that some compounds may exhibit higher anti-panic properties than others, at least in patients with certain features. Patients with PD who experience $35 \% \mathrm{CO}_{2}$ inhalation-induced PAs and who have clinical respiratory symptoms, higher occurrence of spontaneous PAs, and familial loading for PD are thought to be a "respiratory subtype" [45]. In addition, many patients have an irregular breathing pattern during daytime and sleep and a chronic hyperventilation condition [46-48]. A study conducted by our group found that in patients with respiratory $\mathrm{PD}$, fluvoxamine and imipramine have weaker anti-panic properties than sertraline, paroxetine, and clomipramine [49]. Possible explanations are that fluvoxamine has the lowest in vitro anticholinergic effects, which are thought to be involved in respiratory aspects of anti-panic activity [50], whereas imipramine has the weakest effect on the serotonergic system, which plays a crucial role in the pathophysiology of panic-respiration connection $[37,51]$. These findings suggest that when respiratory mechanisms are involved, patients may need alternative interventions. The findings of another study conducted by our group were consistent with the notion of different antipanic properties among SSRIs. A comparison of paroxetine and citalopram in a small sample of patients with PD showed a higher percentage of patients without PAs after 8 weeks of paroxetine treatment (50\%) than citalopram (24\%). Although there was a statistical trend only, this finding suggests higher anti-panic efficacy of paroxetine, possibly related to its adjunctive anticholinergic properties [52]. Finally, a preliminary study has indicated that paroxetine decreases respiratory irregularity in patients with PD, thereby supporting the possibility that this compound may be particularly suitable for patients with respiratory PD [53].

Physical fitness, as measured using cardiopulmonary exercise test, has appeared to be poor in many patients with PD $[54,55]$. Although experimental results are mixed [56-58], aerobic physical exercise may be an adjunctive strategy worthy of consideration in patients who complain of cardiorespiratory symptoms and physical discomfort. Likewise, adjunctive breathing therapies may be useful [59]. 
On the other hand, other alternative interventions may be more suitable for patients with prevalent symptoms involving balance system function. Many patients with PD complain of dizziness, both during and between PAs. Some experimental studies have found subclinical abnormalities in these patients' balance systems and postural instability particularly related to impaired visuovestibular interactions; this feature is thought to influence the development and course of agoraphobia [60-64]. An open study conducted by our group found that 6 weeks of treatment with citalopram facilitated a significant decrease in balance abnormalities, as measured using posturography, in a sample of patients with PD and agoraphobia [60-64]. An open study conducted by our group found that 6 weeks of treatment with citalopram facilitated a significant decrease in balance abnormalities, as measured using posturography, in a sample of patients with PD and agoraphobia [65]. In addition, in our clinical experience, we have observed that patients with PD with agoraphobia and dizziness obtain greater improvement from sertraline than other SSRIs. Although experimental comparisons of different SSRIs are needed to confirm this, we speculate that for agoraphobic patients with PD and chronic dizziness, serotonergic compounds with adjunctive properties on histaminergic (citalopram) and dopaminergic (sertraline) systems may be particularly suitable because both these systems are involved in the regulation of balance function [66, 67]. Finally, preliminary, unpublished data collected by our group suggest that vestibular rehabilitation is beneficial for patients with PD, agoraphobia, and dizziness, who remained symptomatic despite adequate pharmacotherapy with SSRIs and a standard course of CBT. Overall, these findings suggest that patients with this particular symptom profile may benefit from neurotologic examination and alternative integration of pharmacological/non-pharmacological interventions.

In conclusion, in our view, achieving full remission of all panic symptoms, from PAs to physical discomfort, is the crucial step that may allow subsequent recovery of other aspects of PD and complete autonomy in daily life. As described above, this goal may be reached by trying to match patients' symptom profiles and available treatment options based on experimental studies on panic physiopathology. Only when the appropriate "personalized" use of the discussed options fails to achieve panic remission, patients should be considered to be TR, and at that point, clinicians should evaluate the use of nonrecommended compounds with less-established anti-panic effectiveness. Similarly, persistence of anticipatory anxiety/phobic behaviors should be considered as a manifestation of resistance only if these are not caused by the persistence of panic symptoms; if panic symptoms are in fact fully remitted, different cognitive and emotional aspects should be investigated and addressed in CBT sessions or in particular situations via other psychotherapeutic interventions that are not specifically recommended for PD.

It is clear that much more work is required to optimize treatment outcomes in patients with PD. Future large-scale pharmacological/non-pharmacological studies should incorporate biomarker/endophenotype-based approaches, including patterns of neurobiological functions and symptom profiles. This would allow recognition of more suitable targets and intervention outcomes and the evaluation of the effectiveness of therapy according to the specific features of each patient; eventually, this strategy could help overcome the current guidelines, which refer to "average" effects in "average" patients, and facilitate more personalized treatments. Predictive tools can contribute more to 
personalized treatments [68], identifying individual, clinical, biological, and genetic factors, including polymorphisms of genes related to neurotransmitter systems/drug metabolism enzymes [69], that may affect treatment response. Being able to select the most appropriate type and length of therapy for each patient will contribute to increasingly favorable treatment outcomes. In the future, the development and use of predictive tools could decrease the rate of non-responders to available recommended treatments for PD.

\section{Acknowledgements}

The authors would like to thank Alessandra Alciati, MD, and Alice Riva, PsyD, for their contribution to literature search and revision processes.

\section{Compliance with Ethical Standards}

\section{Conflict of Interest \\ Giampaolo Perna declares that he has no conflict of interest. Daniela Caldirola declares that she has no conflict of interest.}

\section{Human and Animal Rights and Informed Consent}

This article does not contain any studies with human or animal subjects performed by any of the authors.

Open Access This article is distributed under the terms of the Creative Commons Attribution 4.0 International License (http://creativecommons.org/licenses/by/4.0/), which permits unrestricted use, distribution, and reproduction in any medium, provided you give appropriate credit to the original author(s) and the source, provide a link to the Creative Commons license, and indicate if changes were made.

\section{References and Recommended Reading}

Papers of particular interest, published recently, have been highlighted as follows:

- Of importance

1. Kessler RC, Petukhova M, Sampson NA, et al. Twelve-month and lifetime prevalence and lifetime morbid risk of anxiety and mood disorders in the united states. Int J Methods Psychiatr Res. 2012;21(3):169-84.

2. Batelaan NM, Van Balkom AJ, Stein DJ. Evidence-based pharmacotherapy of panic disorder: an update. Int J Neuropsychopharmacol. 2012;15(3):403-15.

3. Smit F, Cuijpers P, Oostenbrink J, et al. Costs of nine common mental disorders: Implications for curative and preventive psychiatry. J Ment Health Policy Econ. 2006;9(4):193-200.
4. American Psychiatric Association. Diagnostic and statistical manual of mental disorders (fifth ed.) (DSM-5). Arlington: American Psychiatric Publishing; 2013.

5. American Psychiatric Association. Practice guidelines for the treatment of patients with panic disorder. American Psychiatric Association; 2009. http:// psychiatryonline.org/pb/assets/raw/sitewide/practice_ guidelines/guidelines/panicdisorder.pdf

6. National Institute for Health and Care Excellence (NICE): Generalised anxiety disorder and panic disorder in adults: Management. 2011. https://www.nice. org.uk/guidance/CG113 
7. $\quad$ Perna G, Schruers K, Alciati A, et al. Novel investigational therapeutics for panic disorder. Expert Opin Investig Drugs. 2015;24(4):491-505.

This review, based on preclinical and clinical studies, provides a thorough description of potential novel drugs for panic disorder

8. Baldwin DS, Anderson IM, Nutt DJ, et al. Evidencebased pharmacological treatment of anxiety disorders, post-traumatic stress disorder and obsessivecompulsive disorder: a revision of the 2005 guidelines from the British association for psychopharmacology. J Psychopharmacol. 2014;28(5):403-39.

9.• Freire RC, Zugliani MM, Garcia RF, et al. Treatmentresistant panic disorder: a systematic review. Expert Opin Pharmacother. 2016;17(2):159-68.

This is the previous most recent comprehensive review on treatment-resistant panic disorder

10. Bandelow B, Ruther E. Treatment-resistant panic disorder. CNS Spectr. 2004;9(10):725-39.

11. Perna G, Guerriero G, Caldirola D. Emerging drugs for panic disorder. Expert Opin Emerg Drugs. 2011;16(4):631-45.

12. van Apeldoorn FJ, van Hout WJ, Mersch PP, et al. Is a combined therapy more effective than either CBT or SSRI alone? Results of a multicenter trial on panic disorder with or without agoraphobia. Acta Psychiatr Scand. 2008;117(4):260-70.

13. Caldirola D, Grassi M, Alciati A, et al. Personalized medicine in panic disorder: where are we now? A metaregression analysis. Pers Med Psychiatry. 2017;1-2:26-38.

This study addresses the issue of clinical predictors of pharmacotherapy in PD

14. Baetz M, Bowen RC. Efficacy of divalproex sodium in patients with panic disorder and mood instability who have not responded to conventional therapy. Can J Psychiatr. 1998;43(1):73-7.

15. Hoge EA, Worthington JJ 3rd, Kaufman RE, et al. Aripiprazole as augmentation treatment of refractory generalized anxiety disorder and panic disorder. CNS Spectr. 2008;13(6):522-7.

16. Hirschmann S, Dannon PN, Iancu I, et al. Pindolol augmentation in patients with treatment-resistant panic disorder: a double-blind, placebo-controlled trial. J Clin Psychopharmacol. 2000;20(5):556-9.

17. Dannon PN, Iancu I, Grunhaus L. The efficacy of reboxetine in the treatment-refractory patients with panic disorder: an open label study. Hum Psychopharmacol. 2002;17(7):329-33.

18. Hollifield M, Thompson PM, Ruiz JE, et al. Potential effectiveness and safety of olanzapine in refractory panic disorder. Depress Anxiety. 2005;21(1):33-40.

19. Simon NM, Otto MW, Worthington JJ, et al. Next-step strategies for panic disorder refractory to initial pharmacotherapy: a 3-phase randomized clinical trial. J Clin Psychiatry. 2009;70(11):1563-70.

20. Sepede G, De Berardis D, Gambi F, et al. Olanzapine augmentation in treatment-resistant panic disorder: a 12-week, fixed-dose, open-label trial. J Clin

Psychopharmacol. 2006;26(1):45-9.

21. Pollack MH, Otto MW, Kaspi SP, et al. Cognitive behavior therapy for treatment-refractory panic disorder. J Clin Psychiatry. 1994;55(5):200-5.

22. Heldt E, Blaya C, Isolan L, et al. Quality of life and treatment outcome in panic disorder: cognitive behavior group therapy effects in patients refractory to medication treatment. Psychother Psychosom.

2006;75(3):183-6.

23. Heldt E, Gus Manfro G, Kipper L, et al. One-year follow-up of pharmacotherapy-resistant patients with panic disorder treated with cognitive-behavior therapy: outcome and predictors of remission. Behav Res Ther. 2006;44(5):657-65.

24. Ballenger JC. Clinical guidelines for establishing remission in patients with depression and anxiety. J Clin Psychiatry. 1999;60(Suppl 22):29-34.

25. Bandelow B. Defining response and remission in anxiety disorders: toward an integrated approach. CNS Spectr. 2006;11(10 Suppl 12):21-8.

26. Bandelow B, Baldwin DS, Dolberg OT, et al. What is the threshold for symptomatic response and remission for major depressive disorder, panic disorder, social anxiety disorder, and generalized anxiety disorder? J Clin Psychiatry. 2006;67(9):1428-34.

27. Liberati A, Altman DG, Tetzlaff J, et al. The prisma statement for reporting systematic reviews and metaanalyses of studies that evaluate health care interventions: explanation and elaboration. J Clin Epidemiol. 2009;62(10):e1-34.

28. American Psychiatric Association. Diagnostic and statistical manual of mental disorders (DSM-IV). Washington, DC: Amer Psychiatric Pub; 1994.

29. American Psychiatric Association. Diagnostic and statistical manual of mental disorders fourth edition (text revision) (DSM-IV-TR). Washington, DC: Amer Psychiatric Pub; 2000.

30. International classification of diseases, ninth revision (ICD-9): 1978. http://www.cdc.gov/nchs/icd/icd9.htm

31. International classification of diseases, ninth revision, clinical modification (ICD-9-CM): 1979. http://www. cdc.gov/nchs/icd/icd9cm.htm

32. International classification of diseases, tenth revision (ICD-10): 1994. http://www.cdc.gov/nchs/icd/icd10.htm

33. Goddard AW, Mahmud W, Medlock C, et al. A controlled trial of quetiapine xr coadministration treatment of ssri-resistant panic disorder. Ann Gen Psychiatry. $2015 ; 14-26$.

The sole randomized controlled trial on second-generation antipsychotics in treatment-resistant panic disorder.

34. Payne LA, White KS, Gallagher MW, et al. Second-stage treatments for relative nonresponders to cognitive behavioral therapy (cbt) for panic disorder with or without agoraphobia-continued cbt versus ssri: a randomized controlled trial. Depress Anxiety. 2016;33(5):392-9. 
35. Gloster AT, Sonntag R, Hoyer J, et al. Treating treatment-resistant patients with panic disorder and agoraphobia using psychotherapy: a randomized controlled switching trial. Psychother Psychosom. 2015;84(2):100-9.

36. Perna G, Bertani A, Caldirola D, et al. Modulation of hyperreactivity to $35 \% \mathrm{co} 2$ after one week of treatment with paroxetine and reboxetine: a double-blind, randomized study. J Clin Psychopharmacol. 2004;24(3):277-82.

37. Bertani A, Perna G, Migliarese G, et al. Comparison of the treatment with paroxetine and reboxetine in panic disorder: a randomized, single-blind study. Pharmacopsychiatry. 2004;37(5):206-10.

38. Perna G, Alciati A, Balletta R, et al. Is there room for second-generation antipsychotics in the pharmacotherapy of panic disorder? A systematic review based on prisma guidelines. Int J Mol Sci. 2016;17(4).

A comprehensive systematic review on the use of second generation antipsychotics in panic disorder.

39. Lecrubier Y, Judge R. Long-term evaluation of paroxetine, clomipramine and placebo in panic disorder. Collaborative paroxetine panic study investigators. Acta Psychiatr Scand. 1997;95(2):153-60.

40. Choi KW, Woo JM, Kim YR, et al. Long-term escitalopram treatment in Korean patients with panic disorder: a prospective, naturalistic, open-label, multicenter trial. Clin Psychopharmacol Neurosci.

2012;10(1):44-8.

41. Nardi AE, Freire RC, Mochcovitch MD, et al. A randomized, naturalistic, parallel-group study for the long-term treatment of panic disorder with clonazepam or paroxetine. J Clin Psychopharmacol. 2012;32(1):120-6.

42. Kampman M, Keijsers GP, Hoogduin CA, et al. A randomized, double-blind, placebo-controlled study of the effects of adjunctive paroxetine in panic disorder patients unsuccessfully treated with cognitivebehavioral therapy alone. J Clin Psychiatry. 2002;63(9):772-7.

43. Rodrigues H, Figueira I, Goncalves R, et al. Cbt for pharmacotherapy non-remitters-a systematic review of a next-step strategy. J Affect Disord. 2011;129(1-3):219-28.

44. Perna G, di Pasquale D, Grassi M, et al. Temperament, character and anxiety sensitivity in panic disorder: a high-risk study. Psychopathology. 2012;45(5):300-4.

45. Zugliani MM, Freire RC, Perna G, et al. Laboratory, clinical and therapeutic features of respiratory panic disorder subtype. CNS Neurol Disord Drug Targets. 2015;14(5):627-35.

46. Caldirola D, Bellodi L, Caumo A, et al. Approximate entropy of respiratory patterns in panic disorder. Am J Psychiatry. 2004;161(1):79-87.

47. Grassi M, Caldirola D, Di Chiaro NV, et al. Are respiratory abnormalities specific for panic disorder? A meta-analysis. Neuropsychobiology. 2014;70(1):52-60.
48. Grassi M, Caldirola D, Vanni G, et al. Baseline respiratory parameters in panic disorder: a meta-analysis. J Affect Disord. 2013;146(2):158-73.

49. Perna G, Bertani A, Caldirola D, et al. Antipanic drug modulation of $35 \% \mathrm{co} 2$ hyperreactivity and short-term treatment outcome. J Clin Psychopharmacol. 2002;22(3):300-8.

50. Battaglia M, Bertella S, Ogliari A, et al. Modulation by muscarinic antagonists of the response to carbon dioxide challenge in panic disorder. Arch Gen Psychiatry. 2001;58(2):114-9.

51. Perna G, Caldirola D, Bellodi L. Panic disorder: From respiration to the homeostatic brain. Acta Neuropsychiatr. 2004;16(2):57-67.

52. Perna G, Bertani A, Caldirola D, et al. A comparison of citalopram and paroxetine in the treatment of panic disorder: a randomized, single-blind study. Pharmacopsychiatry. 2001;34(3):85-90.

53. Yeragani VK, Rao R, Tancer M, et al. Paroxetine decreases respiratory irregularity of linear and nonlinear measures of respiration in patients with panic disorder. A preliminary report. Neuropsychobiology. 2004;49(2):53-7.

54. Caldirola D, Namia C, Micieli W, et al. Cardiorespiratory response to physical exercise and psychological variables in panic disorder. Rev Bras Psiquiatr. 2011;33(4):385-9.

55. Muotri RW, Bernik MA. Panic disorder and exercise avoidance. Rev Bras Psiquiatr. 2014;36(1):68-75.

56. Broocks A, Bandelow B, Pekrun G, et al. Comparison of aerobic exercise, clomipramine, and placebo in the treatment of panic disorder. Am J Psychiatry. 1998;155(5):603-9.

57. Gaudlitz K, Plag J, Dimeo F, et al. Aerobic exercise training facilitates the effectiveness of cognitive behavioral therapy in panic disorder. Depress Anxiety. 2015;32(3):221-8.

58. Bartley CA, Hay M, Bloch MH. Meta-analysis: Aerobic exercise for the treatment of anxiety disorders. Prog Neuro-Psychopharmacol Biol Psychiatry. 2013;45:34-9.

59. Meuret AE, Wilhelm FH, Ritz T, et al. Feedback of endtidal pco 2 as a therapeutic approach for panic disorder. J Psychiatr Res. 2008;42(7):560-8.

60. Caldirola D, Teggi R, Bondi S, et al. Is there a hypersensitive visual alarm system in panic disorder? Psychiatry Res. 2011;187(3):387-91.

61. Teggi R, Caldirola D, Bondi S, et al. Vestibular testing in patients with panic disorder and chronic dizziness. Acta Otorhinolaryngol Ital. 2007;27(5):243-7.

62. Teggi R, Caldirola D, Colombo B, et al. Dizziness, migrainous vertigo and psychiatric disorders. J Laryngol Otol. 2010;124(3):285-90.

63. Perna G, Dario A, Caldirola D, et al. Panic disorder: The role of the balance system. J Psychiatr Res. 2001;35(5):279-86.

64. Coelho CM, Balaban CD. Visuo-vestibular contributions to anxiety and fear. Neurosci Biobehav Rev. 2015;48:148-59. 
65. Perna G, Alpini D, Caldirola D, et al. Serotonergic modulation of the balance system in panic disorder: an open study. Depress Anxiety. 2003;17(2):101-6.

66. Lacour M. Betahistine treatment in managing vertigo and improving vestibular compensation: clarification. J Vestib Res. 2013;23(3):139-51.

67. Chabbert C. Principles of vestibular pharmacotherapy. Handb Clin Neurol. 2016;137:207-18.
68. Perna G, Grassi M, Caldirola D, Nemeroff CB. The revolution of personalized psychiatry: will technology make it happen sooner? Psychol Med. 2017;1-9. https://doi.org/10.1017/S0033291717002859.

69. Caldirola D, Perna G. Is there a role for pharmacogenetics in the treatment of panic disorder? Pharmacogenomics. 2015;16(8):771-4. 